Associer semi-conducteurs

et supraconducteurs est une

idée a priori surprenante, tant

les propriétés électroniques

de ces deux états sont opposées.

Néanmoins, le couplage entre

les électrons et les modes de

vibration du réseau atomique,

à l'origine de la supraconduc-

tivité conventionnelle, peut

être très fort dans les semi-

conducteurs du groupe IV.

Leur caractère semi-conducteur est dû au fait que les électrons

sont impliqués dans les liaisons

covalentes qui assurent

la cohésion du matériau

et n'interviennent pas dans

la conductivité électrique.

En rendant ces liaisons

covalentes conductrices,

on obtient une nouvelle

famille de supraconducteurs.

La température critique des

matériaux covalents atteint

aujourd'hui $39 \mathrm{~K}$ dans $\mathrm{MgB}_{2}$,

mais pourrait largement

dépasser cette valeur dans

certains composés carbonés.

\section{Les semi-conducteurs supraconducteurs du groupe IV}

Thierry Klein ${ }^{(1)}$ (thierry.klein@grenoble.cnrs.fr), Xavier Blase ${ }^{(1)}$, Christophe Marcenat ${ }^{(2)}$,

Claude Chapelier ${ }^{(2)}$ et Étienne Bustarret ${ }^{(1)}$

(1) Institut Néel, CNRS et Université Joseph Fourier, BP 166, 38042 Grenoble Cedex.

(2) SPSMS, UMR-E CEA / UJF Grenoble 1, INAC, 38054 Grenoble.

\section{Historique}

Lorsqu'il s'agit de supraconductivité, l'attention est souvent focalisée sur les records de température critique $\left(T_{c}\right)$ et la compréhension des mécanismes " non conventionnels " conduisant à ces températures élevées. Néanmoins, le domaine de la supraconductivité traditionnelle, véhiculée par l'interaction entre électrons et vibrations du réseau (les phonons) et décrite par la théorie de Bardeen, Cooper et Schrieffer (BCS) [1], traverse périodiquement des périodes de jouvence liées à la synthèse de nouveaux matériaux, ou à la mise en évidence d'une transition électronique particulière dans des matériaux déjà connus. L'idée qu'un semi-conducteur dopé puisse devenir supraconducteur avait déjà fait son chemin dans les années 1960. La supraconductivité fut même observée à l'époque dans $\mathrm{SrTiO}_{3}$ et $\mathrm{Ge}_{1-x} \mathrm{Te}$, mais elle ne suscita que peu d'intérêt, du fait rieures à $0,5 \mathrm{~K}$ [2]. Au contraire, la découverte spectaculaire, en 2001, de la supraconductivité de $\mathrm{MgB}_{2}$, avec $T_{\mathrm{c}} \sim 39 \mathrm{~K}$, soit près de deux fois le record établi par que les températures critiques étaient infé-
$\mathrm{Nb}_{3} \mathrm{Ge}$ en $1973\left(T_{\mathrm{c}} \sim 23 \mathrm{~K}\right)$, relança ducteurs conventionnels.

Au-delà de la faible masse des atomes de bore, qui conduit à des fréquences de phonons élevées favorisant ainsi une haute $T_{c}$, la valeur exceptionnelle de $39 \mathrm{~K}$ est la conséquence directe de la contribution à la conduction électronique des liaisons covalentes (notées $\sigma$ ) du bore (liaisons planaires de type $\mathrm{sp}^{2}$, voir les schémas $\mathrm{A}$ et $\mathrm{B}$ de la figure 1). $\mathrm{MgB}_{2}$ cristallise dans une structure hexagonale constituée de feuillets de bore (fig. 1c, p. 5 ; fig. 5 , p. 8 ), avec une structure en nid d'abeilles équivalente à celle du graphite (fig. 1a), séparés par les atomes de Mg. Dans le graphite, la bande électronique formée par les liaisons $\sigma$ assure la cohésion des plans et est située bien en dessous de l'énergie de Fermi $E_{\mathrm{F}}$ (fig. 2a). Par conséquent, la conduction n'est pas assurée par les électrons $\sigma$, mais uniquement par le recouvrement des orbitales $p_{z}$ (liaisons $\pi$ perpendiculaires aux plans) des atomes d'un même plan ou de plans adjacents. Dans $\mathrm{MgB}_{2}$, la présence des ions $\mathrm{Mg}^{2+}$ entre les feuillets de bore conduit à un décalage important des énergies, jusqu'à sérieusement l'intérêt pour les supracon-

\section{Couplage fort entre électrons et phonons}

encadré 1

Dans la théorie de Bardeen, Cooper et Schrieffer (BCS) [1, 3], la température critique $T_{\mathrm{C}}$ est celle au-dessus de laquelle la densité de quasi-particules (paires de Cooper thermiquement dissociées) devient trop importante pour permettre un condensat supraconducteur stable. Si cette densité dépend de la valeur de la bande interdite supraconductrice $\Delta$ (ou gap), $\Delta$ est lui-même déterminé de manière auto-cohérente par la densité de quasi-particules. La température critique, $T_{\mathrm{C}}=1,13 h v_{\mathrm{D}} \exp \left(-1 / N\left(E_{\mathrm{F}}\right) V\right)$, est donc celle à laquelle $\Delta$ est réduit à zéro.

L'exposant $-1 / N\left(E_{\mathrm{F}}\right) V$ provient de I'approximation de BCS, valable pour $N\left(E_{\mathrm{F}}\right) V<<1$, où I'interaction électron-phonon est une constante $V$ pour des électrons dont l'énergie est comprise dans une bande de largeur $h v_{\mathrm{D}}$ autour de $E_{\mathrm{F}}$, et nulle autrement. $v_{\mathrm{D}}$ est une fréquence typique des vibrations du réseau cristallin.

Cette approximation ne se justifie pas pour les matériaux covalents, où le couplage électronphonon $\lambda_{\text {el-ph }}$ est " fort ». $\lambda_{\text {el-ph }}$ est alors obtenu comme une intégrale sur les fréquences des phonons pertinents. Après insertion dans la théorie BCS, on a :

$T_{\mathrm{C}}=1,13 h v_{\mathrm{D}} \exp \left[-\left(1+\lambda_{\mathrm{el}-\mathrm{ph}}\right) /\left(\lambda_{\mathrm{el}-\mathrm{ph}} \mu^{*}\right)\right]$, ou $\mu^{*}$ est le potentiel de Coulomb " écranté ». Dans la limite de couplage faible, on retrouve $\lambda_{\text {el-ph }} \sim N\left(E_{\mathrm{F}}\right) V$. 


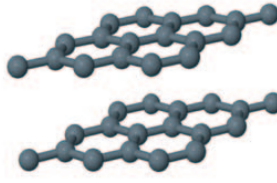

Graphite

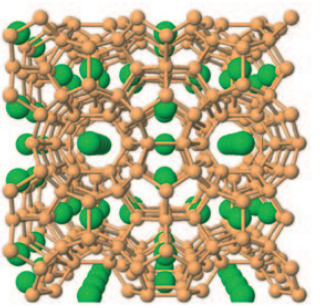

Ba@Si-46

$T_{c}=8 \mathrm{~K}$

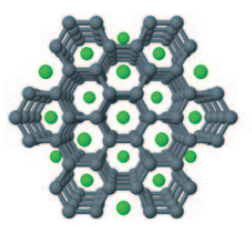

$\mathrm{CaC}_{6}$

$T_{c}=11,5 \mathrm{~K}$

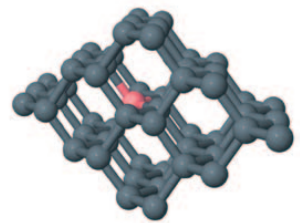

Diamant dopé

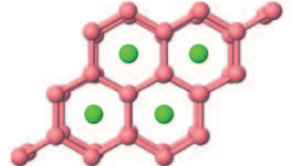

$\mathrm{MgB}_{2}$

C $T_{\mathrm{c}}=39 \mathrm{~K}$

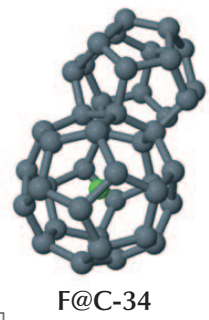

$T=77 \mathrm{~K}(?)$

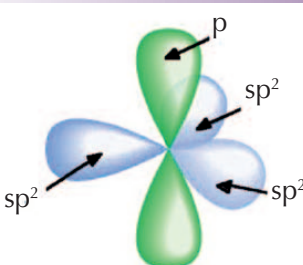

A

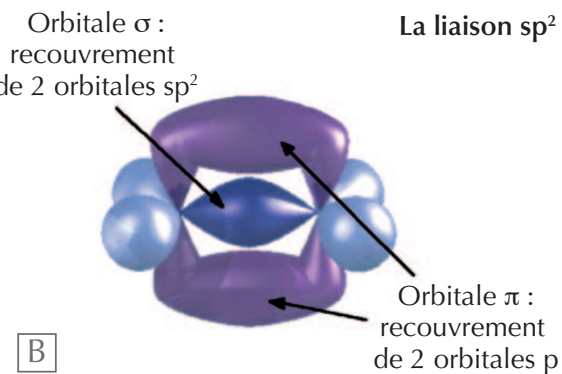

ramener le haut de la bande $\sigma$ au-dessus de $E_{\mathrm{F}}$ (fig. 2c). La conduction se trouve alors assurée à la fois par la bande $\pi$ et par la bande $\sigma$. Comme elles sont intimement liées à l'alignement planaire précis des atomes $\mathrm{B}$ (ou $\mathrm{C}$ ), les orbitales $\sigma$ sont très "sensibles " aux vibrations de ces atomes. Par conséquent, elles donnent lieu à un couplage électron-phonon $\lambda_{\text {el-ph }}$ particulièrement fort et à une $T_{c}$ " anormalement " élevée (encadré 1 ). $\mathrm{MgB}_{2}$ est ainsi le premier exemple d'une supraconductivité "multiple ", forte en ce qui concerne les électrons de la bande $\sigma$, mais faible pour les électrons de la bande $\pi$.

Cependant, $\mathrm{MgB}_{2}$ n'est pas le premier exemple de système covalent supraconducteur ayant une $T_{\mathrm{c}}$ (relativement) élevée. En effet, en 1995, une température critique de l'ordre de $8 \mathrm{~K}$ avait été découverte dans les clathrates de silicium dopés au baryum [4]. Les clathrates sont construits à partir de cages de silicium $\mathrm{Si}_{\mathrm{n}}$ (avec $\left.\mathrm{n}=20,24,28\right)$, qui partagent certaines de leurs faces (atomes marron de la figure 1d). Les atomes Si y conservent leur configuration tétraédrique $\mathrm{sp}^{3}$, formant des liaisons covalentes très directionnelles et localisées entre les atomes premiers voisins. C'est l'analogue en trois dimensions $\left(\mathrm{sp}^{3}\right)$ des liaisons planaires $\left(\mathrm{sp}^{2}\right)$ de $\mathrm{MgB}_{2}$. Dans les deux cas, les liaisons $\sigma$ assurent la rigidité structurale et un couplage électron-phonon efficace. Cependant, les clathrates de silicium sont semi-conducteurs, avec une bande interdite de l'ordre de 1,8 eV. Il faut introduire des « impuretés » dans ces matériaux (les " doper») de manière à les rendre conducteurs. C'est la structure même des clathrates qui le permet, en autorisant une intercalation aisée d'atomes de baryum aux centres des cages (atomes verts de la figure $1 \mathrm{~d}$ ). $\mathrm{Ba}_{8} \mathrm{Si}_{46}$ fut ainsi le premier semi-conducteur de la colonne IV présentant une supraconductivité à plusieurs kelvins.
1. Principales structures des supraconducteurs covalents. (a) La structure graphitique du carbone est composée de feuillets hexagonaux. (b) Entre ces plans de carbone (gris) peuvent être intercalés des éléments comme Ca ou Y (verts). (c) MgB 2 a une structure cristallographique analogue, pour laquelle les plans B (roses) sont séparés par le Mg (vert) (voir aussi la figure 5, p. 8). (d) Les clathrates de Si sont des structures en cage ( $\mathrm{Si}_{46}$, marron), au centre desquelles sont insérés des atomes de Ba (verts). (e) Le diamant peut être dopé par substitution (par exemple au bore, atome rose) jusqu'à obtenir des alliages de type B-C pour lesquels une très forte valeur de $T_{\mathrm{c}}$ a été prédite. (f) De même, des hautes $T_{\mathrm{c}}$ ont été prédites dans les clathrates de carbone.

Dans le graphite (a), la conduction électrique est assurée par les bandes $\pi$ (voir schémas A et B) auxquelles s'ajoute, dans le cas du graphite intercalé (b), une conduction par la bande $\zeta$. Dans les autres cas, les liaisons covalentes $\sigma$ jouent un rôle fondamental, soit en structure planaire $\left[s p^{2},(c)\right]$, soit en structure tridimensionnelle $\left[s p^{3},(d-f)\right]$.

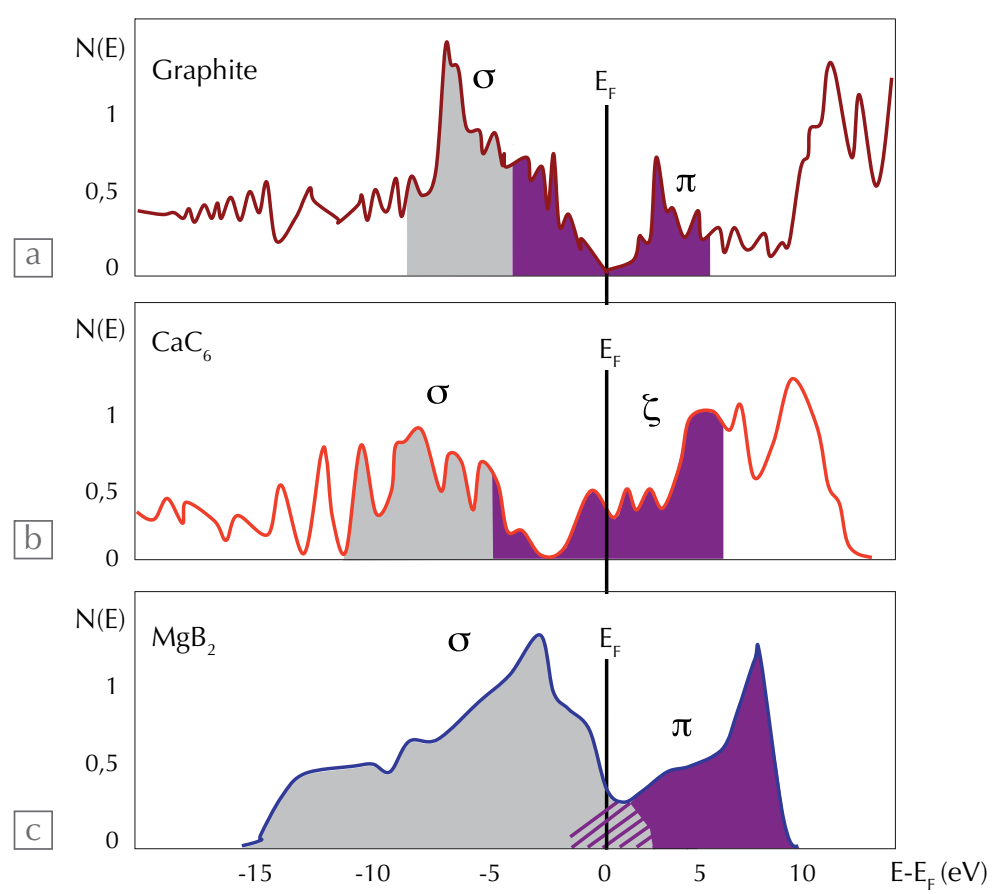

2. Densité d'états électroniques $N(E)$ en fonction de l'énergie $E$, pour le graphite $(\mathrm{a}), \mathrm{CaC}_{6}$ (b) et $\mathrm{MgB}_{2}$ (c). Dans ce diagramme, le niveau de Fermi $E_{F}$ correspond à l'énergie du dernier niveau électronique occupé à $T=0 \mathrm{~K}$. Seuls les électrons proches de $E_{\mathrm{F}}$ contribuent aux propriétés électroniques et thermiques du solide : la densité $N\left(E_{\mathrm{F}}\right)$ des états électroniques à $E_{\mathrm{F}}$ joue donc un rôle fondamental. Notamment, elle intervient directement dans le coefficient de couplage entre électrons et phonons, $\lambda_{\mathrm{e}-\mathrm{ph}}=N\left(E_{\mathrm{F}}\right) V$, où $V$ est un potentiel d'interaction traduisant la force du couplage. (a) Dans le graphite, les niveaux électroniques formés par les liaisons $\sigma$ (en gris) se trouvent en dessous de $E_{\text {F. }}$ La conductivité est déterminée par le minimum de densité d'états au milieu des niveaux $\pi$ (en mauve). (b) Dans $\mathrm{CaC}_{6}$, l'énergie de Fermi est déplacée dans les niveaux dits $\zeta$, hybrides entre les liaisons $\pi$ du C et les niveaux du Ca. (c) Enfin, dans $\mathrm{MgB}_{2}$, le niveau de Fermi se trouve dans la bande $\sigma$. 


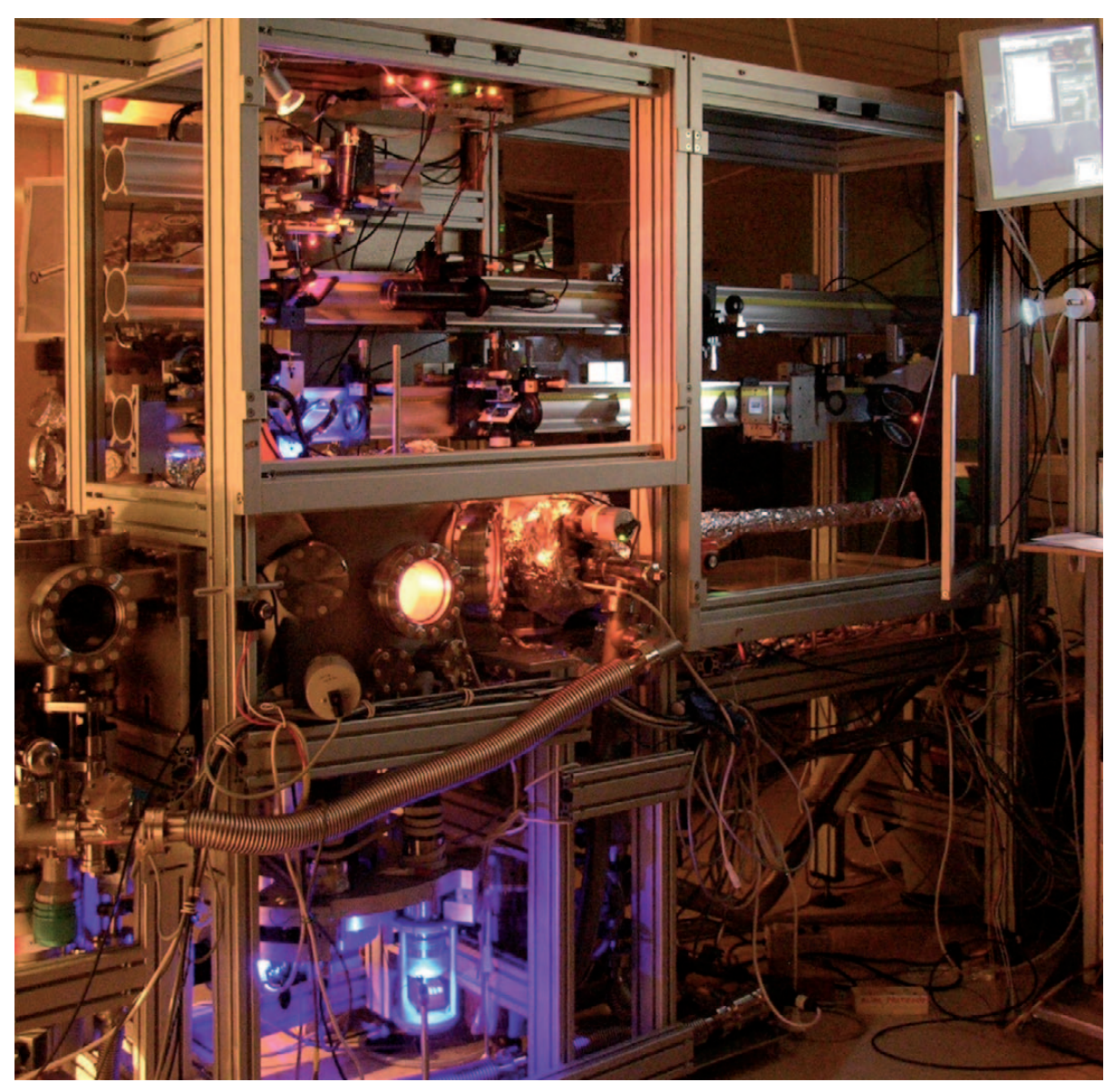

3. Procédé de dopage induit par laser.

Ci-dessus : vue d'ensemble du bâti d'épitaxie laser sous ultravide de l'Institut d'Électronique Fondamentale d'0rsay. Courtoisie : Dominique Debarre.

Ci-dessous : principe de la technique de dépôt GILD (Gas Immersion Laser Doping), dans laquelle un gaz contenant le dopant (atomes de bore) est adsorbé sur la surface d'un substrat de silicium (a), qui est ensuite fondu par laser pendant quelques dizaines de nanosecondes (b). La recristallisation obtenue par refroidissement extrêmement rapide $\left(\sim 10^{10} \mathrm{~K} / \mathrm{s}\right)$ permet de piéger les atomes de bore en sites substitutionnels (bien au-delà de la limite de solubilité thermodynamique de B dans Si), tout en conservant une bonne reprise d'épitaxie sur le substrat (c).

Laser pulsé

- Atomes B

- Atomes Si
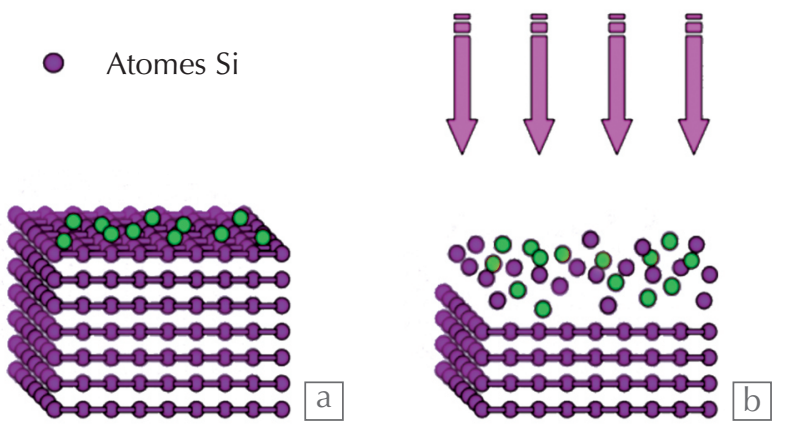

\section{\〉 \\ Lorsque diamant et silicium deviennent supraconducteurs.}

Outre sa structure de type graphite (hybridation $\mathrm{sp}^{2}$ ), il est bien connu que le carbone peut cristalliser dans la structure diamant ( $\mathrm{sp}^{3}$, fig. 1e). Le diamant et le silicium, qui ont des structures cristallographiques équivalentes, sont probablement les systèmes covalents les plus connus. Le premier, car il est le prototype des isolants et le matériau le plus dur qui soit, et le second parce qu'il est le constituant principal de l'électronique moderne. Or, la question de savoir si ces composés peuvent également être supraconducteurs n'avait pas reçu de réponse jusqu'à un passé récent.

La relation empirique déjà évoquée entre covalence, rigidité, et ampleur de $\lambda_{\text {el-ph }}$ entraîna la rencontre de deux communautés distinctes : celle travaillant sur les matériaux ultra-durs et celle s'intéressant à la supraconductivité.

Ainsi, au début des années 2000, le diagramme de phases ternaire $\mathrm{C}: \mathrm{B}: \mathrm{N}$ faisait l'objet de nombreuses études visant à élaborer des systèmes de très haute dureté. En 2004, deux groupes réussirent à synthétiser sous haute pression des carbures de bore avec, en sous-produit, du diamant contenant environ 2 à 3\% (atomiques) de bore (en substitution sur les sites du C), un taux de dopage suffisant pour rendre le diamant conducteur. Ces travaux établirent que, lorsqu'il devient métallique, le diamant dopé est également supraconducteur en dessous de 4 K [5]. Aujourd'hui, du diamant supraconducteur polycristallin, monocristallin et même nanocristallin a été obtenu sous haute pression, ou par des techniques de dépôt en phase vapeur. La température critique a été poussée jusqu'à environ $10 \mathrm{~K}$.

En 2006, un groupe français a montré [6] que le silicium pouvait, lui aussi, devenir supraconducteur (avec une $T_{\mathrm{c}}$ maximale de l'ordre de $1 \mathrm{~K}$ ), lorsqu'il contient environ 2 à $8 \%$ de bore. Or, ce taux de dopage se situe bien au-delà de la limite de solubilité du bore dans le silicium. Il ne peut être atteint que grâce à des techniques de croissance hors équilibre, comme celle de "Gas Immersion Laser Doping", qui est décrite dans la figure 3 .

Plus récemment, on a découvert que le carbure de silicium $\mathrm{SiC}$ et le germanium deviennent eux aussi supraconducteurs (avec des $T_{\mathrm{c}} \sim 1 \mathrm{~K}$ ), lorsqu'ils sont respectivement dopés au bore et au gallium. 
Doper un isolant jusqu'à ce qu'il devienne métallique semble une façon peu directe pour fabriquer un bon supraconducteur. Comme mentionné dans l'introduction, le principal attrait des systèmes covalents est le grand $\lambda_{\text {el-ph }}$, proportionnel au produit $N\left(E_{\mathrm{F}}\right) V$. De ce point de vue, le diamant dopé est exceptionnel : le potentiel attractif entre électrons, $V$, est $50 \%$ plus élevé que dans $\mathrm{MgB}_{2}$. Il est donc surprenant que la température critique la plus élevée mesurée dans le diamant $(\sim 10 \mathrm{~K})$, soit nettement plus faible que celle de $\mathrm{MgB}_{2}$. Cela provient des valeurs très différentes des densités d'états électroniques au niveau de Fermi, $N\left(E_{\mathrm{F}}\right)$, et donc des taux très différents d'électrons participant à la supraconductivité. Si, en dimension deux, $N\left(E_{\mathrm{F}}\right)$ est indépendante de la densité électronique $n$, elle croît comme $\sim n^{1 / 3}$ en dimension trois. $N\left(E_{\mathrm{F}}\right)$ est donc élevée dans le composé lamellaire $\mathrm{MgB}_{2}$, mais modeste dans les matériaux tridimensionnels pour des taux de dopage pertinents. Les calculs ab initio [7] réalisés pour le carbone, le silicium et le carbure de silicium dopés avec quelques pourcents de bore, montrent que ces matériaux ont en effet des valeurs de $\boldsymbol{\lambda}_{\text {e-ph }}$ comprises entre 0,3 et 0,5 (au lieu de $\sim 1$ dans $\mathrm{MgB}_{2}$ ), ce qui conduit à des températures critiques de quelques kelvins, en bon accord avec les résultats expérimentaux.

\section{Vers de nouveaux systèmes à haute température critique?}

Si l'on s'inspire de la théorie de BCS (encadré 1 ), une grande valeur de $\boldsymbol{\lambda}_{\mathrm{e}-\mathrm{ph}}$ (et donc de $T_{\mathrm{c}}$ ) requiert des matériaux mariant une forte densité d'états $N\left(E_{\mathrm{F}}\right)$ avec un bon potentiel d'interaction $V$. On rappelle que $V$ augmente lorsque la masse de l'atome diminue (si l'on passe du silicium au carbone, par exemple), car les fréquences typiques des phonons sont alors plus élevées. De plus, des calculs ont montré que $V$ est plus important pour une hybridation de type $\mathrm{sp}^{3}$ (plutôt que $\mathrm{sp}^{2}$ ). La limitation des composés supraconducteurs covalents tridimensionnels est donc la faible valeur de $N\left(E_{\mathrm{F}}\right)$

En ce qui concerne le diamant, des travaux pour accroître $N\left(E_{\mathrm{F}}\right)$ sont en cours, soit en optimisant le processus de dopage chimique, soit en utilisant d'autres modes d'injection des porteurs [8]. Cependant, les expériences montrent que le lien entre la concentration de bore et la température critique n'est pas trivial (encadré 2, p. 8). Au-delà de quelques pourcents de bore, $T_{\mathrm{c}}$ sature, probablement du fait de l'agrégation du bore en paires électriquement inactives ou à son incorporation sur des sites interstitiels.

De nombreuses pistes restent à explorer. Par exemple, il a été prédit que $\mathrm{BC}_{5}$ en structure cubique aurait une $T_{c}$ équivalente à celle de $\mathrm{MgB}_{2}$. Cette structure a été synthétisée à haute pression et haute température, mais la nature nanocristalline du matériau obtenu n'a pas permis la mesure d'éventuelles propriétés supraconductrices.

D'autres pistes vers les hautes $T_{\mathrm{c}}$ sont les matériaux avec une structure en "cage ", comme les fullerènes de carbone dopés en métaux alcalins. En effet, les "boules » de $\mathrm{C}_{60}$ se répartissent sur un réseau cubique pour former un " cristal moléculaire " ayant des bandes d'énergie très étroites, associées à de fortes densités d'états ${ }^{(a)}$. Dans le fullérène $\mathrm{C}_{60}$ non dopé, ces bandes sont soit vides, soit totalement remplies : le solide est isolant. Le dopage peut être effectué en intercalant des éléments alcalins dans les cavités laissées libres entre les boules, ce qui conduit à une $T_{\mathrm{c}}$ de l'ordre de 30 K. Si l'on "profite» ici pleinement des fortes valeurs de la densité d'états, des études théoriques ont montré qu'il reste pas mal de choses à faire du côté du potentiel $V$. Dans $\mathrm{C}_{60}$, les liaisons sont principalement de type $\mathrm{sp}^{2}$, mais $V$ augmente rapidement quand on passe de $\mathrm{C}_{60}$ à $\mathrm{C}_{20}$ (b)

Les clathrates de silicium, déjà mentionnés en introduction, constituent d'autres exemples de structures en cage, dans lesquelles, contrairement aux fullerènes, les atomes ont chacun quatre premiers voisins et toutes les liaisons de type $\mathrm{sp}^{3}$ sont satisfaites. Les bandes électroniques sont plus étroites que dans le diamant, et donnent donc lieu à de fortes densités d'états. Enfin, leur structure permet des taux de dopage élevés, ainsi qu'une grande flexibilité dans le choix du dopant. En particulier, dans le clathrate dopé au baryum noté Ba $@$ Si $_{46}$ (fig. 1d), on obtient une $T_{c}$ d'environ $8 \mathrm{~K}$, à comparer à $0,6 \mathrm{~K}$ observé dans le silicium cubique dopé au bore. Malheureusement, les fréquences typiques des phonons, et donc $T_{\mathrm{c}}$, sont ici limitées par la masse élevée des atomes de Si. Malgré des prédictions théoriques encourageantes et de nombreuses tentatives, aucun clathrate de carbone (fig. 1f) n'a été synthétisé à ce jour.
Terminons par les composés de structure graphitique [9], comme $\mathrm{CaC}_{6}$ ou $\mathrm{YbC}_{6}$ (fig. 1b). Si le niveau de Fermi ne se situe pas dans la bande $\sigma$, l'hybridation entre les niveaux électroniques $\pi$ du carbone et ceux de l'intercalant ( $\mathrm{Ca}$ ou $\mathrm{Yb})$, regroupés sous le terme de "bande $\zeta$ " (fig. 2b), conduit à une $T_{\mathrm{c}}$ relativement élevée (jusqu'à 11,5 K). Il est alors tentant de chercher des matériaux pour lesquels le niveau de Fermi croise à la fois la bande $\sigma$ et la bande $\zeta$. Des valeurs de $T_{c}$ atteignant $150 \mathrm{~K}$ ont ainsi été prédites pour $\mathrm{Li}_{x} \mathrm{BC}_{8}(x<1)$, mais n'ont pas été observées à ce jour.

\section{Applications potentielles}

Le silicium dopé au bore jouera peut-être un rôle important dans le développement de dispositifs à base de jonctions supraconducteur-normal-supraconducteur (SNS). Quand la région normale $(\mathrm{N})$ est un semiconducteur, le fort désaccord entre les densités électroniques conduit à une discontinuité du potentiel chimique dans la jonction. Cette discontinuité se traduit par une barrière dite "de Schottky ", qui limite considérablement le transfert des paires de Cooper à travers la jonction, et donc la fonctionnalité de tels dispositifs. La supraconductivité du silicium "ultradopé » rendrait possible la fabrication des deux régions à partir du même matériau, en changeant simplement le niveau de dopage. On éviterait ainsi les barrières de Schottky à l'interface. Dès lors, le contrôle du courant traversant la jonction par la grille capacitive influant sur la densité électronique dans la partie normale devient possible, ce qui ouvrirait la voie à la fabrication du transistor Josephson à effet de champ (JOFET, voir figure 4).

\〉

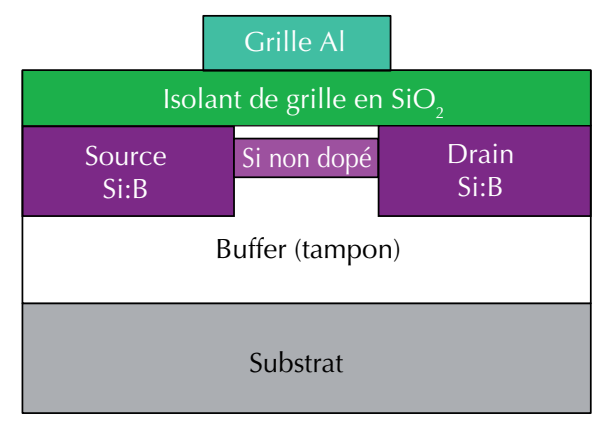

4. Transistor Josephson à effet de champ (JOFET). L'utilisation d'un même matériau pouvant être rendu soit supraconducteur soit isolant, par dopage, et I'application d'une tension de grille simplifieraient beaucoup la conception de ce genre de dispositif. 
>>

La découverte de la supraconductivité du silicium, du SiC et du diamant dopés ouvre également maintes possibilités pour la conception de dispositifs électromécaniques de taille nanométrique pour la détection de forces, masses, charges ou déplacements, avec une résolution telle que l'on pourrait mesurer la quantification de ces grandeurs. L'absence de dissipation électrique (sans couche métallique supplémentaire à déposer sur le résonateur) augmente le facteur de qualité et la sensibilité du système. De nouveaux effets pourraient être observés, qui proviendraient de la nature cohérente de la supraconductivité.

En particulier, les extraordinaires propriétés physiques, électroniques et thermiques du diamant en font un matériau unique pour le développement de nanosystèmes électromécaniques. Son module de Young inégalé permettra de réaliser des poutres ayant des fréquences de résonance $v$ bien plus élevées $(\mathrm{GHz})$ que celles des résonateurs actuels, rendant la limite quantique accessible à des températures envisageables $\left(\sim 40 \mathrm{mK}<h v / k_{\mathrm{B}}\right)$. La poutre peut être excitée en faisant circuler un courant en présence d'un champ magnétique - la force de Lorentz conduit alors à l'oscillation du dispositif. La $T_{\mathrm{c}}$ et le champ critique supérieur [3] de l'ordre de quelques teslas, tous deux supérieurs à ceux des supraconducteurs habituellement utilisés, permettront de travailler à des champs magnétiques élevés, où le couplage électromécanique est plus fort.

\section{Conclusion}

En conclusion, bien que le mécanisme d'appariement semble conventionnel, c'està-dire décrit par l'interaction électronphonon dans le cadre de la théorie BCS, la supraconductivité induite par dopage dans les semi-conducteurs covalents est un développement inattendu et prometteur pour la recherche actuelle et future.

(a) Toutes les bandes électroniques contiennent le même nombre d'états. Donc, plus une bande est étroite en énergie - on dit qu'elle est moins dispersive - plus la densité d'états devient grande.

(b) C'est-à-dire, lorsque l'on augmente le caractère $\mathrm{sp}^{3}$ de l'hybridation du carbone. De même, il est possible d'augmenter $V$ dans les nanotubes de carbone en les confinant dans des canaux de zéolite. $V$ croît alors lorsque le diamètre se réduit, entraînant une augmentation de $T_{\mathrm{c}}$ jusqu'à $14 \mathrm{~K}$ pour des tubes de $0,4 \mathrm{~nm}$.

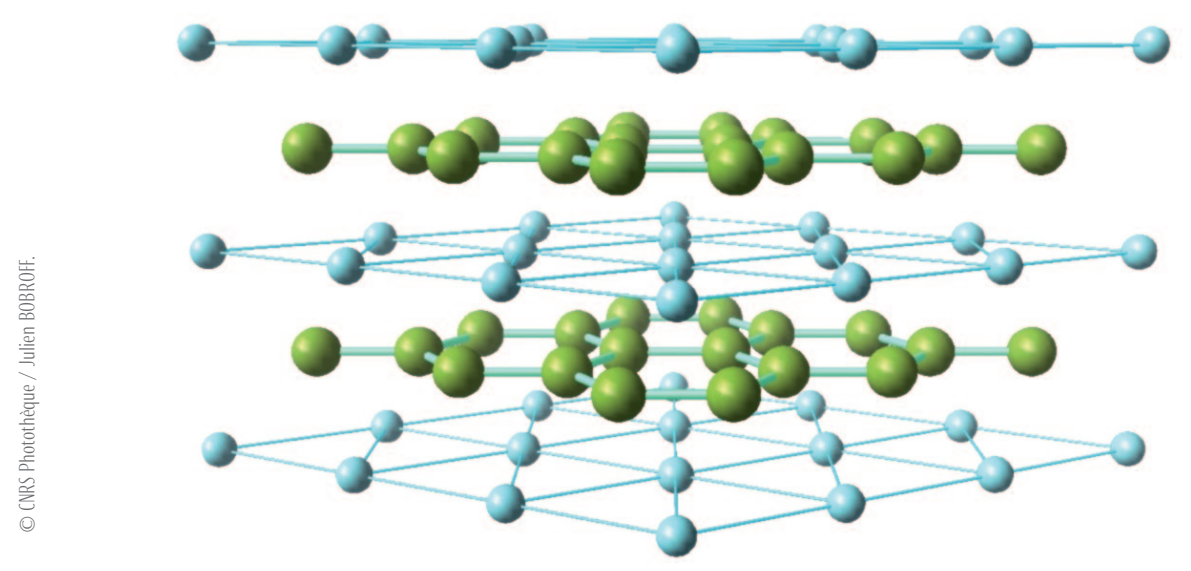

5. Structure cristallographique du diborure de magnésium $\mathrm{MgB}_{2}$. Les atomes de bore sont en vert, ceux de magnésium en bleu.

\section{Des électrons proches de la localisation} encadré 2

Un aspect fondamental des semi-conducteurs supraconducteurs est leur faible densité de porteurs. De ce fait, ces systèmes sont proches d'une transition métal-isolant (TMI). À ceci s'ajoute l'effet du désordre inhérent au dopage, notamment le piégeage d'électrons dans des états localisés, néfaste pour la supraconductivité. Néanmoins, dans le diamant dopé, $T_{\mathrm{C}}$ reste anormalement élevée lorsque I'on se rapproche de la TMI [10] (fig. 6), alors que la théorie BCS prévoit qu'elle décroît exponentiellement lorsque $\lambda_{\mathrm{e}-\mathrm{ph}} \sim N\left(E_{\mathrm{F}}\right) V$ tend vers zéro.

La situation est a priori plus simple dans le cas du silicium dopé, pour lequel la supraconductivité est obtenue pour des taux de dopage de l'ordre de $2 \%$ à $5 \%$, bien supérieurs à la valeur correspondant à la TMI $(\sim 0,01 \%)$. Or, les calculs ab initio [8] de $\lambda_{\text {el-ph }}$ suggèrent là aussi une variation très rapide de $T_{\mathrm{C}}$ en fonction du dopage, tandis que les expériences montrent encore une fois une décroissance "lente » de $T_{C}$.

Le rôle du désordre reste donc à élucider.

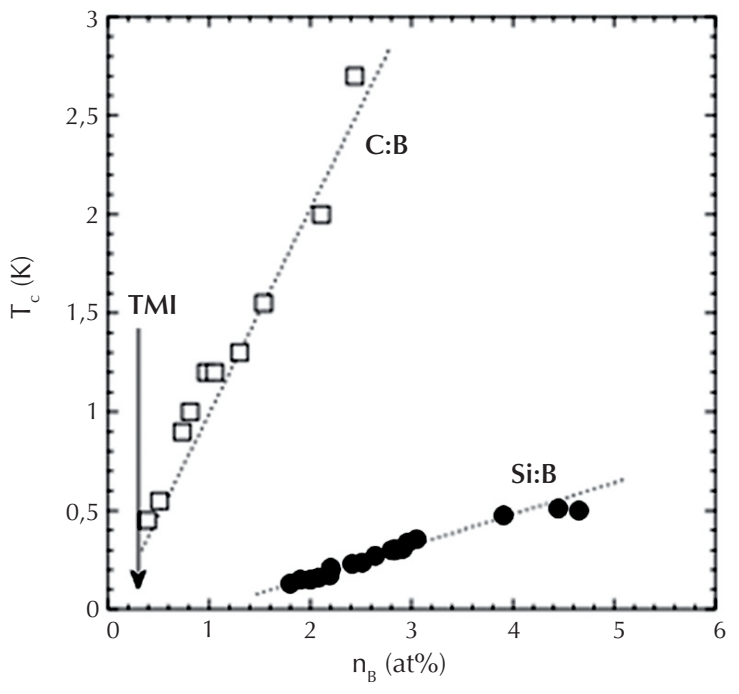

6. Évolution de la température critique $\left(T_{C}\right)$ en fonction du taux de dopage en bore $\left(n_{\mathrm{B}}\right)$ dans le diamant (C:B) et le silicium (Si:B).

\section{Références}

1• P. Nozières, Reflets de la Physique 7 (2007), p. 14.

2•X. Blase, É. Bustarret, C. Chapelier, T. Klein et C. Marcenat, Nature Materials, 8 (2009), p. 275 et références incluses.

3• K. van der Beek, Reflets de la Physique 27 (2011), p. 6.

4• H. Kawaji et al., Phys. Rev. Lett. 74 (1995) 1427 ; D. Connétable et al., Phys. Rev. Lett. 91 (2003) 247001.

5• E.A. Ekimov et al., Nature 428 (2004) 542 ;

É. Bustarret et al., Phys. Rev. Lett. 93 (2004) 237005.

6• É. Bustarret et al., Nature 444 (2006) 465.
7• L. Boeri et al., Phys. Rev. Lett. 93 (2004) 237002 ; K.W. Lee et al., Phys. Rev. Lett. 93 (2004) 237003; X. Blase et al., Phys. Rev. Lett. 93 (2004) 237004.

8• W.E. Pickett, Physica C 468 (2008) 126 ; J.E. Moussa et M.L. Cohen, Phys. Rev. B 77 (2008) 064518 ; M. Calandra et F. Mauri, Phys. Rev. Lett. 101 (2008) 016401.

9• T.E. Weller et al., Nature Phys. 1 (2005) 39.

10• T. Klein et al., Phys. Rev. B 75 (2007) 165313 ; C. Marcenat et al., Phys. Rev. B 81 (2010) 020501(R). 